\title{
Robotic sacrocolpopexy for pelvic organ prolapse: Nationwide costs, utilization and outcomes ninety days following surgery
}

\author{
Lannah L Lua ${ }^{1 *}$, Prathamesh Pathak ${ }^{2}$ and Vani Dandolu ${ }^{2}$ \\ ${ }^{1}$ Department of Obstetrics and Gynecology, University of Nevada Las Vegas School of Medicine, Las Vegas, Nevada, USA \\ ${ }^{2}$ OptumCare Women's Health, Las Vegas, Nevada, USA
}

\begin{abstract}
Introduction: Robotic sacrocolpopexy (RSC) has increased dramatically over the last decade. Nationally representative cost estimates of robotic surgery for apical prolapse have yet to be reported. The study determined index and follow-up costs and healthcare utilization up to ninety days after RSC.

Methods: Retrospective cohort study was conducted using Truven MarketScan data from 2008 to 2012. Patients were categorized based on route of index procedure and performance of concomitant hysterectomy. Cohorts included RSC ( $\mathrm{N}=2,006)$, laparoscopic sacrocolpopexy (LSC) (N=10,708), abdominal sacrocolpopexy (ASC) $(\mathrm{N}=6,126)$, and vaginal colpopexy (VC) $(\mathrm{N}=45,808)$. Index and follow-up costs were calculated using inpatient, outpatient, emergency, and pharmacy services data. Healthcare utilization was determined from readmissions, emergency room visits, and outpatient visits. T-test and z-test were utilized for statistical analyses.

Results: Mean index cost of RSC was $\$ 15,821$ without and $\$ 17,288$ with hysterectomy, while mean follow-up cost was $\$ 19,250$ without and $\$ 19,847$ with hysterectomy. Higher index and follow-up costs were incurred from RSC compared to ASC, VC, and LSC ( $<<0.00001)$. Approximately $3.44 \%$ and $10.42 \%$ of RSC patients had at least one readmission and at least one emergency room visit, respectively. Mean number of outpatient visits was $4.7 \pm 4.3$. Higher rates of emergency room visits occurred following RSC than $\mathrm{VC}$ without hysterectomy (11.44\% vs. $10.70 \%$, $\mathrm{p}=0.03)$. More outpatient visits were associated with $\mathrm{RSC}$ than $\mathrm{VC}$ with hysterectomy ( $4.5 \pm 4.1$ vs. $4.2 \pm 3.9$ visits, $\mathrm{p}=0.01)$ and LSC overall $(4.7 \pm 4.3$ vs. $4.4 \pm 4.1$ visits, $\mathrm{p}=0.007)$.
\end{abstract}

Conclusions: RSC is associated with higher costs and healthcare utilization during the first ninety days.

\section{Brief summary}

Healthcare costs and utilization during the first ninety days after surgery are higher in patients undergoing robotic sacrocolpopexy compared to other apical prolapse procedures.

Abbreviations: ASC: Abdominal Sacrocolpopexy; CPT-4: Current Procedural Terminology, $4^{\text {th }}$ edition; HMO: Health Maintenance Organization; ICD-9: International Statistical Classification of Diseases and Related Health Problems, $9^{\text {th }}$ revision; LSC: Laparoscopic Sacrocolpopexy; POP: Pelvic Organ Prolapse; PPO: Preferred Provider Organization; RSC: Robotic Sacrocolpopexy; VC: Vaginal Colpopexy.

\section{Introduction}

Pelvic organ prolapse (POP) is becoming increasingly prevalent due to the aging population in the United States. The number of women suffering from POP is projected to increase by $46 \%$ over the next 40 years, from 3.3 million to 4.9 million [1]. Consequently, the cumulative incidence of POP surgery has increased to $12.6 \%$, representing over 220,000 women undergoing surgery for symptomatic POP in the United States annually [2-3]. Reoperations occur in approximately $30 \%$ of these patients [2]. Current estimates indicate the total annual cost for POP surgery to be over $\$ 1$ billion [3]. These statistics highlight the importance of employing a cost-effective procedure for surgical management of symptomatic POP. The current literature, however, is too scant to adequately indicate which approach should be recommended [4].

Abdominal sacrocolpopexy (ASC) is considered the gold standard surgical treatment of apical prolapse. However, minimally invasive surgery for POP, specifically robotic sacrocolpopexy (RSC), has increased dramatically over the last decade in excess of $25 \%$ annually [4-5]. Considerable growth in RSC is partly attributed to its advantages over conventional laparoscopic sacrocolpopexy (LSC), such as enhanced three-dimensional visualization, physiologic tremor filtering, improved dexterity, and increased freedom of movement of instruments [2, 6-9]. Reduced morbidity associated with RSC compared to ASC, including decreased blood loss, decreased postoperative pain, shorter hospitalization and faster recovery, has also led to its widespread integration into POP surgery [10-11].

${ }^{*}$ Correspondence to: Lannah L. Lua, M.D, 1701 W Charleston Blvd, Suite 290 Las Vegas, Nevada 89102; Tel: +1 (702) 671-2385; Fax: +1 (702) 671-2333; E-mail: lannah.lua@gmail.com

Key words: apical prolapse, healthcare costs, healthcare utilization, pelvic organ prolapse, robotic surgery, sacrocolpopexy

Received: May 20, 2019; Accepted: May 27, 2019; Published: May 29, 2019 
Despite these reported advantages, cost justification poses a major limitation to the adoption of a robotic approach to sacrocolpopexy. Costanalysis studies comparing RSC with other approaches show conflicting results. Two small randomized controlled trials demonstrated increased expenses incurred from RSC compared to LSC $[6,12]$. RSC was shown to produce the highest hospital charges, being $16 \%$ and $47 \%$ more expensive than LSC and ASC, respectively [13]. Other studies, on the other hand, found RSC to be associated with lower costs [14-15]. Furthermore, the calculated cost of RSC varies widely among studies, ranging from $\$ 6,668$ to $\$ 16,278$ [12,14-16]. These studies are limited by small sample size, experience of a single surgeon or institution, data from a few surgical centers, and/or inclusion only of the cost of the procedure itself.

Nationally representative cost estimates of robotic surgery in POP in the United States have yet to be reported. The purpose of this study is to determine nationwide costs of RSC, including costs associated with the index procedure and the follow-up period up to ninety days, and compare these costs to other approaches to apical prolapse surgery. Secondary analyses assessed nationwide utilization and outcomes associated with RSC compared to other approaches up to ninety days after surgery, including inpatient readmissions, emergency room visits, outpatient visits, length of stay, complications and post-operative procedures.

\section{Materials and methods}

Retrospective cohort study was conducted utilizing data from the Truven MarketScan Commercial Claims and Encounter database from 2008 to 2012. The database consists of approximately 138 million unique patients that are representative of the privately insured population in the United States and specifically include active employees, early retirees, Consolidated Omnibus Budget Reconciliation Act continuers, and their dependents. All patients are less than 65 years of age and are insured by over 100 employer-sponsored plans. The study was determined to fall outside of review by the Institutional Review Board, as the database contains deidentified data.

Female patients between the age of 18 and 65 years, who underwent surgery for apical prolapse from 2008 to 2012 and were continuously enrolled during the index date and the follow-up period, were included in the study. Apical prolapse procedures of interest were identified using the Current Procedural Terminology, $4^{\text {th }}$ edition (CPT-4) coding system. The index date represents the date of the patient's first apical prolapse procedure, while the follow-up period represents the 89 days following the index procedure. Patients were categorized based on the route of their index procedure. Study groups include ASC, vaginal colpopexy (VC), LSC, and RSC. Each group was further stratified depending on whether the procedure involved concomitant hysterectomy. Any patient with more than one index procedure was categorized in multiple study groups and analyzed separately.

Baseline characteristics (age, comorbidities, type of insurance plan, and geographic region) were collected. Direct costs for the index procedure and the follow-up period were calculated using the total payment variable and included costs incurred from inpatient, outpatient, emergency room, and pharmacy services. Indirect costs, productivity losses, and other societal costs were not included in the calculations. Patients with costs equating to zero (i.e. stale dated claim), a negative value, or three standard deviations above the cohort mean were excluded from the cost calculations to obtain unbiased estimates. The dollar value for all cost estimates was inflation-adjusted to the mid- year estimate for 2014 using the medical component of the consumer price index.

Healthcare resource utilization was derived from data on inpatient readmissions, emergency room visits, and outpatient services during the follow-up period. Other secondary endpoints, including complications, length of stay, and additional procedures that were performed during the follow-up period, were also analyzed for each study group. Complications were identified using the $9^{\text {th }}$ revision of the International Statistical Classification of Diseases and Related Health Problems (ICD-9) codes. Additional procedures during the follow-up period were identified using CPT- 4 codes.

The data were analyzed using descriptive statistics, $\mathrm{t}$-test and $\mathrm{z}$-test. Continuous variables were expressed as mean values with standard deviations, while categorical variables were expressed as frequencies or percentages. The Student's t-test was utilized for comparison of means, and the z-test was used for checking the equality of two proportions. The RSC cohort was assigned as the reference group for all comparisons. A p-value of less than 0.05 was considered to be statistically significant.

\section{Results}

A total of 115,589 POP procedures were identified in the database between 2008 and 2012. Among reported apical prolapse procedures, 6,126 cases were abdominal (ASC), 45,808 cases were vaginal (VC), and 10,708 cases were laparoscopic. In the latter cohort, 8,702 cases were performed via conventional laparoscopy (LSC), while 2,006 cases involved robotic assistance (RSC). The mean age of patients who underwent surgery for apical prolapse was $51.4 \pm 8.9$ years, with $42.7 \%$ of the population falling in the 55 to 65 age group. Most individuals were insured under a preferred provider organization (PPO) plan with an overall rate of $64.3 \%$, followed by a health maintenance organization (HMO) plan at $10.6 \%$. Individuals from the Southern region of the United States were overrepresented and comprised the majority of the population (44.2\%), followed by the North Central region (22.7\%). The most common pre-index comorbidities were hypertension $(31.5 \%)$, depression (13.7\%), and diabetes (9.2\%). Baseline characteristics for each study cohort are summarized in Table 1.

Study groups were further stratified according to performance of concurrent hysterectomy. Approximately similar rates of all POP procedures were performed without hysterectomy $(52 \%, \mathrm{~N}=60,175)$ and with hysterectomy $(48 \%, \mathrm{~N}=55,414)$. Slightly more abdominal $(58 \%, \mathrm{~N}=3,571)$ and vaginal $(52 \%, \mathrm{~N}=23,771)$ apical prolapse procedures involved concomitant hysterectomy. However, the difference was considerably larger when looking at laparoscopic and robotic procedures, with hysterectomy concomitantly performed in $70 \%(\mathrm{~N}=6,051)$ of LSC and $65 \%(\mathrm{~N}=1,307)$ of RSC cases. Concurrent hysterectomy also raised the cost of the procedure for all routes. Therefore, study endpoints for individual cohorts are shown for both with hysterectomy and without hysterectomy.

The number of RSC cases increased from 40 to 640 cases over five years, comprising $3.27 \%$ of minimally-invasive abdominal sacrocolpopexies in 2008 and up to $24.38 \%$ in 2012 . The five-year mean index cost of RSC was calculated to be $\$ 15,821$ without hysterectomy and $\$ 17,288$ with hysterectomy (Figure 1 ). This overall index cost was significantly higher compared to that of ASC, VC, and LSC whether or not the procedure involved concomitant hysterectomy (Figure 1). Compared to RSC without hysterectomy, the mean index cost difference was $\$ 3,420$ for ASC (95\% CI [ $\$ 2,537, \$ 4,303], \mathrm{p}<0.00001), \$ 5,802$ for 
Table 1. Demographic characteristics of patients who underwent apical prolapse procedures by route

\begin{tabular}{|c|c|c|c|c|c|c|c|c|}
\hline & \multicolumn{2}{|c|}{ Overall } & \multicolumn{2}{|c|}{ Abdominal } & \multicolumn{2}{|c|}{ Vaginal } & \multicolumn{2}{|c|}{ Laparoscopic } \\
\hline & $\mathbf{N}$ & $\%$ & $\mathbf{N}$ & $\%$ & $\mathbf{N}$ & $\%$ & $\mathbf{N}$ & $\%$ \\
\hline No. of patients & \multicolumn{2}{|c|}{62,642} & \multicolumn{2}{|c|}{6,126} & \multicolumn{2}{|c|}{45,808} & \multicolumn{2}{|c|}{10,708} \\
\hline \multicolumn{9}{|l|}{ Age (years) } \\
\hline Mean & \multicolumn{2}{|c|}{51.4} & \multicolumn{2}{|c|}{51.3} & \multicolumn{2}{|c|}{51.6} & \multicolumn{2}{|c|}{50.6} \\
\hline $\mathrm{SD}$ & \multicolumn{2}{|c|}{8.9} & \multicolumn{2}{|c|}{8.9} & \multicolumn{2}{|c|}{9.0} & \multicolumn{2}{|c|}{8.9} \\
\hline \multicolumn{9}{|l|}{ Age group (years) } \\
\hline $18-44$ & 14,658 & $23.4 \%$ & 1,359 & $22.8 \%$ & 10,509 & $22.9 \%$ & 2,720 & $25.4 \%$ \\
\hline $45-54$ & 21,286 & $34.0 \%$ & 2,092 & $35.1 \%$ & 15,301 & $33.4 \%$ & 3,866 & $36.1 \%$ \\
\hline $55-65$ & 26,748 & $42.7 \%$ & 2,509 & $42.1 \%$ & 19,998 & $43.7 \%$ & 4,122 & $38.5 \%$ \\
\hline \multicolumn{9}{|l|}{ Plan type } \\
\hline HMO & 6,649 & $10.6 \%$ & 662 & $11.1 \%$ & 4,876 & $10.6 \%$ & 1,094 & $10.2 \%$ \\
\hline POS & 4,578 & $7.3 \%$ & 502 & $8.4 \%$ & 3,307 & $7.2 \%$ & 730 & $6.8 \%$ \\
\hline PPO & 40,295 & $64.3 \%$ & 3,808 & $63.9 \%$ & 29,782 & $65.0 \%$ & 6,701 & $62.6 \%$ \\
\hline Missing & 4,586 & $7.3 \%$ & 352 & $5.9 \%$ & 3,265 & $7.1 \%$ & 940 & $8.8 \%$ \\
\hline Other & 6,577 & $10.5 \%$ & 638 & $10.7 \%$ & 4,578 & $10.0 \%$ & 1,243 & $11.6 \%$ \\
\hline \multicolumn{9}{|l|}{ Region } \\
\hline Northeast & 8,434 & $13.5 \%$ & 966 & $16.2 \%$ & 5,470 & $11.9 \%$ & 1,821 & $17.0 \%$ \\
\hline North Central & 14,247 & $22.7 \%$ & 1,343 & $22.5 \%$ & 10,506 & $22.9 \%$ & 2,320 & $21.7 \%$ \\
\hline South & 27,680 & $44.2 \%$ & 2,706 & $45.4 \%$ & 20,692 & $45.2 \%$ & 4,413 & $41.2 \%$ \\
\hline West & 10,587 & $16.9 \%$ & 757 & $12.7 \%$ & 7,915 & $17.3 \%$ & 1,870 & $17.5 \%$ \\
\hline Unknown & 1,694 & $2.7 \%$ & 188 & $3.2 \%$ & 1,225 & $2.7 \%$ & 284 & $2.7 \%$ \\
\hline \multicolumn{9}{|l|}{ Comorbidities } \\
\hline Diabetes & 5,741 & $9.2 \%$ & 578 & $9.7 \%$ & 4,290 & $9.4 \%$ & 835 & $7.8 \%$ \\
\hline Heart disease & 2,339 & $3.7 \%$ & 238 & $4.0 \%$ & 1,766 & $3.9 \%$ & 311 & $2.9 \%$ \\
\hline Hypertension & 19,731 & $31.5 \%$ & 1,937 & $32.5 \%$ & 14,641 & $32.0 \%$ & 3,020 & $28.2 \%$ \\
\hline Cerebrovascular disease & 1,342 & $2.1 \%$ & 137 & $2.3 \%$ & 1,009 & $2.2 \%$ & 185 & $1.7 \%$ \\
\hline Depression & 8,598 & $13.7 \%$ & 846 & $14.2 \%$ & 6,247 & $13.6 \%$ & 1,403 & $13.1 \%$ \\
\hline
\end{tabular}

SD standard deviation, HMO health maintenance organization, POS point of service, PPO preferred provider organization

VC (95\% CI [ $\$ 5,028, \$ 6,576], \mathrm{p}<0.00001)$, and $\$ 3,034$ for LSC (95\% CI $[\$ 2,161, \$ 3,907], \mathrm{p}<0.00001)$. Compared to RSC with hysterectomy, the mean index cost difference was $\$ 4,266$ for ASC $(95 \%$ CI $[\$ 3,635, \$ 4,897]$, $\mathrm{p}<0.00001), \$ 5,712$ for $\mathrm{VC}(95 \% \mathrm{CI}[\$ 5,165, \$ 6,259], \mathrm{p}<0.00001)$, and $\$ 4,301$ for LSC (95\% CI $[\$ 3,710, \$ 4,892], \mathrm{p}<0.00001)$.

In terms of follow-up costs, the calculated mean cost of RSC was $\$ 19,250$ without hysterectomy and $\$ 19,847$ with hysterectomy (Figure 1). Follow-up costs associated with RSC were likewise significantly higher compared to that of ASC, VC, and LSC with or without hysterectomy (Figure 1). Compared to RSC without hysterectomy, the mean followup cost difference was $\$ 3,072$ for ASC (95\% CI [\$1,841, \$4,303], $\mathrm{p}<0.00001), \$ 6,210$ for VC $(95 \%$ CI $[\$ 5,132, \$ 7,288], \mathrm{p}<0.00001)$, and $\$ 2,855$ for LSC (95\% CI $[\$ 1,652, \$ 4,058], \mathrm{p}<0.00001)$. Compared to RSC with hysterectomy, the mean follow-up cost difference was $\$ 4,461$ for $\operatorname{ASC}(95 \%$ CI $[\$ 3,706, \$ 5,216], \mathrm{p}<0.00001), \$ 5,703$ for $\mathrm{VC}(95 \%$ CI $[\$ 5,065, \$ 6,341], \mathrm{p}<0.00001)$, and $\$ 3,744$ for LSC $(95 \%$ CI $[\$ 3,038$, $\$ 4,450], \mathrm{p}<0.00001)$.

Data on healthcare resource utilization during the follow-up period, including inpatient readmissions, emergency room visits and outpatient visits, are illustrated in Figure 2. During the 5-year period, the rate of at least one inpatient readmission within ninety days following RSC was 3.44\% (3.60\% without hysterectomy, 3.40\% with hysterectomy). The readmission rate following RSC was significantly lower than that following LSC with hysterectomy (3.40\% vs. $5.00 \%$, $95 \%$ CI $[-0.027,-0.005], \mathrm{p}=0.01)$ and LSC overall (3.44\% vs. $4.67 \%, 95 \%$ CI $[-0.021 \%,-0.003 \%], \mathrm{p}=0.02)$. Readmissions following other apical prolapse procedures were comparable to that of RSC (Figure 2).

The rate of at least one emergency room visit within ninety days following RSC was $10.42 \%$ (11.44\% without hysterectomy, 9.87\% with hysterectomy). These rates were comparable to that of other apical prolapse procedures, except for VC without hysterectomy (Figure 2). A higher proportion of emergency room visits was observed following RSC without hysterectomy compared to VC without hysterectomy (11.44\% vs. $10.70 \%, 95 \%$ CI [0\%, 0.056\%], $\mathrm{p}=0.03)$.

The mean number of outpatient visits within 90 days following RSC was $4.7 \pm 4.3$ (5.0 4.5 visits without hysterectomy, $4.5 \pm 4.1$ visits with hysterectomy). Statistically significant differences in number of outpatient visits were observed following VC, LSC and all POP procedures in comparison to RSC (Figure 2). More outpatient visits were associated with RSC than with VC with hysterectomy $(4.5 \pm 4.1$ vs. $4.2 \pm 3.9$ visits, $\mathrm{p}=0.01)$, LSC overall $(4.7 \pm 4.3$ vs. $4.4 \pm 4.1$ visits, $\mathrm{p}=0.007)$, all POP procedures with hysterectomy ( $4.5 \pm 4.1$ vs. $4.2 \pm 3.9$ visits, $\mathrm{p}=0.01)$, and all POP procedures overall ( $4.7 \pm 4.3$ vs. $4.5 \pm 4.2$ visits, $\mathrm{p}=0.007)$. 


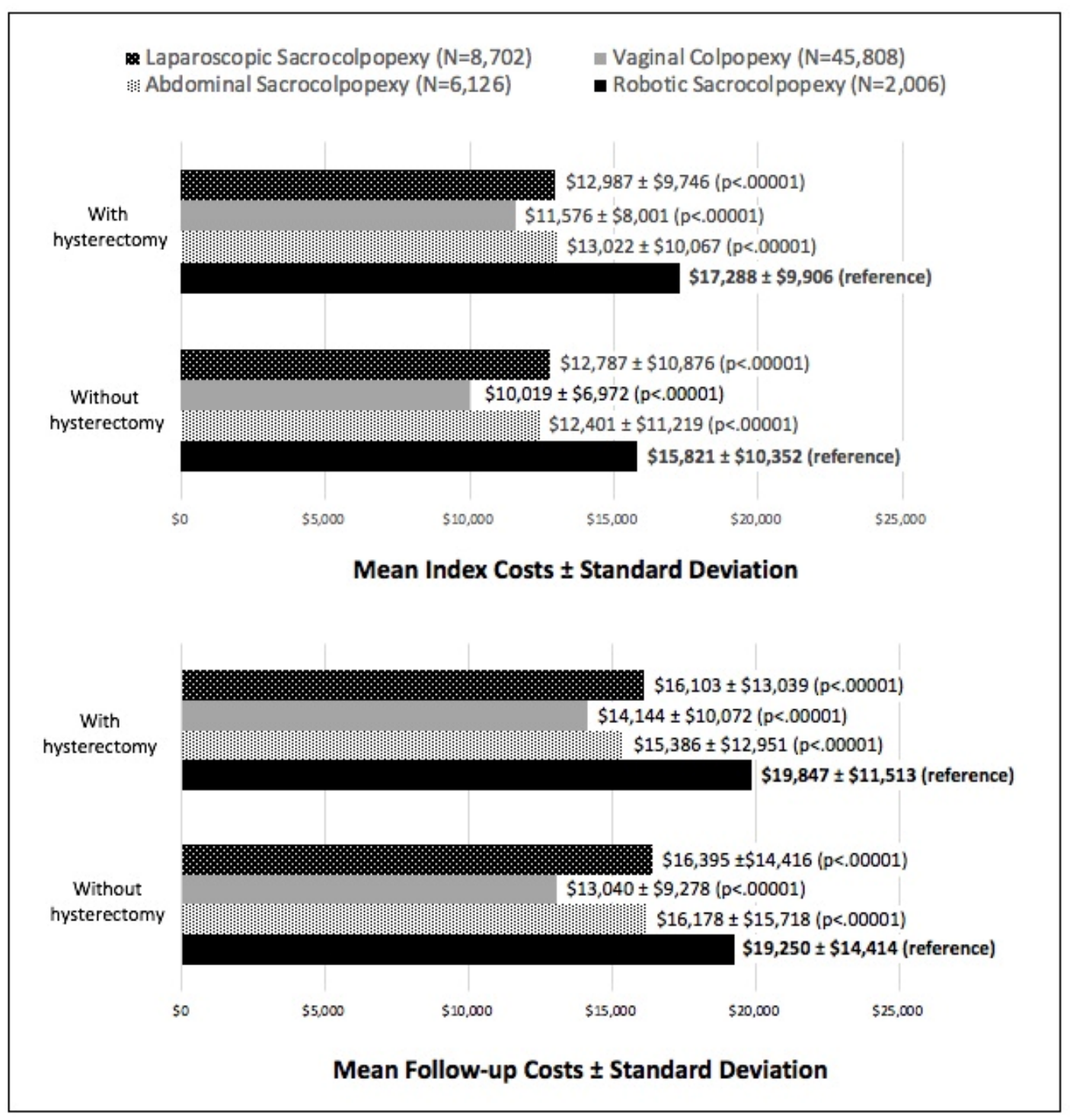

Figure 1. 90-day cost of apical prolapse procedures (2008-2012)

Over 60 different complications occurring during the follow-up period were analyzed. Only those complications that were relevant to an apical prolapse procedure and/or statistically significant are listed in Table 2. When evaluating complications potentially relating to a sacrocolpopexy, significantly higher rates of complications generally occurred following RSC compared to other approaches. Ureteral stricture or kinking (ICD-9 593.3) occurred in $0.43 \%(\mathrm{~N}=3)$ of RSC patients compared to $0.16 \%(\mathrm{~N}=4)$ of ASC patients $(\mathrm{p}=0.008)$. Meshrelated complications (ICD-9 996.76) were observed in 6.58\% $(\mathrm{N}=86)$ of RSC patients, compared to $4.48 \%(\mathrm{~N}=155)$ of ASC patients $(\mathrm{p}<0.001)$. Other potentially relevant complications, including dyspareunia and injury to the bowel and bladder, were not statistically significant (Table 2).

Among the different procedures performed post-operatively during the follow-up period, higher rates were generally observed following RSC. Statistically significant and/or relevant procedures are listed in Table 2. Of note, significant differences were observed only when the apical prolapse procedure was performed with hysterectomy. Removal of prosthetic graft (CPT-4 57295, 57296, $57426)$ was performed in $0.46 \%(\mathrm{~N}=6)$ of RSC patients, compared to $0.20 \%(\mathrm{~N}=7)$ of ASC patients $(\mathrm{p}=<0.001)$ and $0.20 \%(\mathrm{~N}=12)$ of LSC patients $(\mathrm{p}=0.016)$. Destruction of vaginal lesion (CPT-4 57061) was performed in $0.23 \%(\mathrm{~N}=3)$ of RSC patients, compared to $0.08 \%(\mathrm{~N}=5)$ of LSC patients $(\mathrm{p}=0.019)$. Revision of ureter $(\mathrm{CPT}-450700,50715)$ occurred in $0.46 \%(\mathrm{~N}=6)$ of RSC patients, compared to $0.07 \%(\mathrm{~N}=12)$ of VC patients $(\mathrm{p}<0.001)$. Performing an exploratory laparotomy in the post-operative period (CPT-4 49000) occurred in $0.23 \%(\mathrm{~N}=3)$ of RSC patients, compared to $0.04 \%(\mathrm{~N}=7)$ of VC patients $(\mathrm{p}<0.001)$. Statistically significant differences were not observed in other relevant procedures, including blood transfusion, percutaneous nephrostomy tube placement, ureteroneocystotomy, and repair of bladder wound (Table 2).

Mean length of stay with RSC was $1.45 \pm 0.94$ days $(1.47 \pm 0.85$ days without hysterectomy, $1.44 \pm 0.99$ days with hysterectomy). When compared to other apical prolapse procedures, no statistically significant differences were observed. Mean length of stay was $2.51 \pm 1.91$ days for 


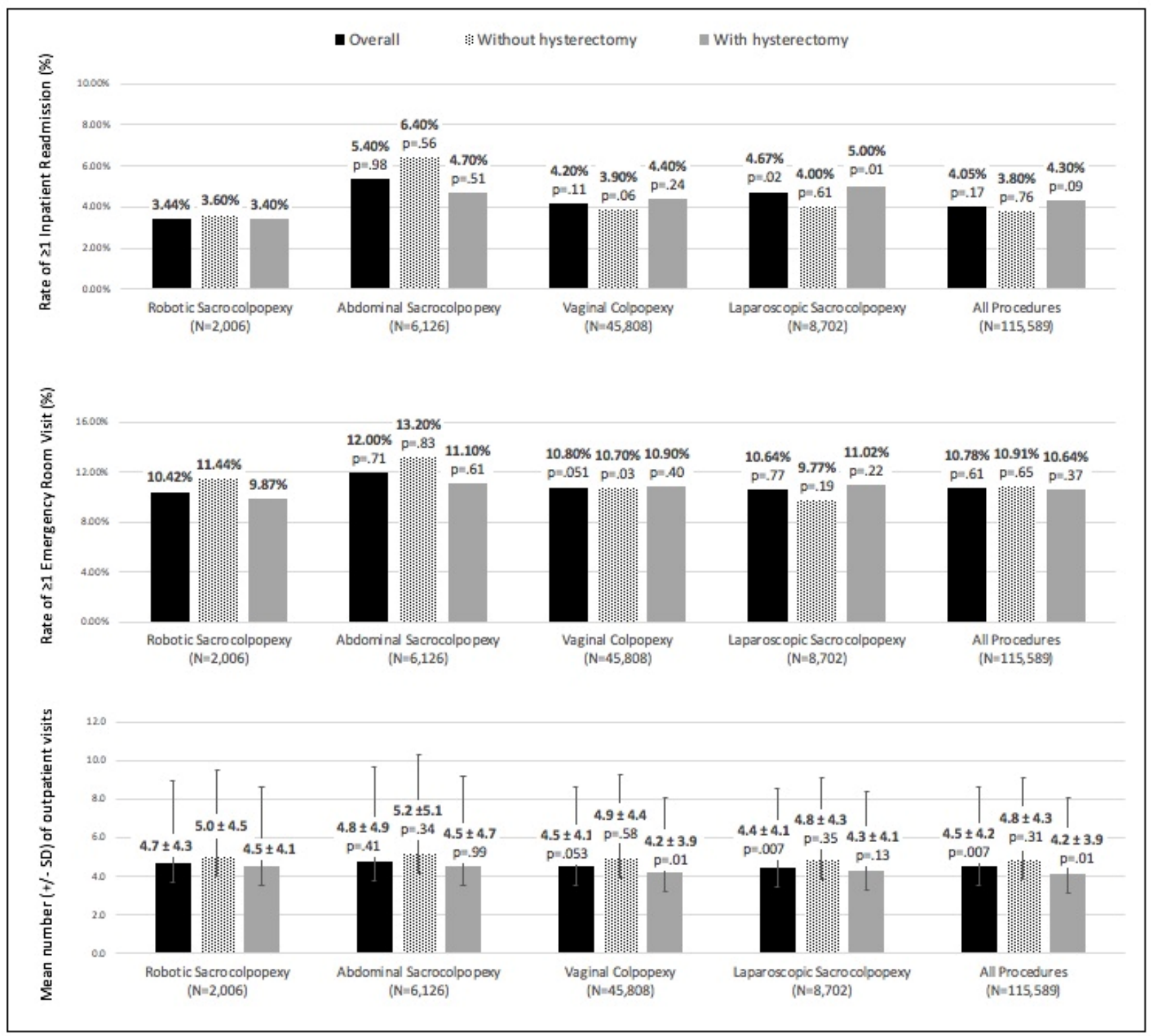

Figure 2. Inpatient readmissions, emergency room visits, and outpatient visits during the 90-day follow-up period (2008-2012)

ASC, $1.60 \pm 0.94$ days for VC, and $1.51 \pm 1.14$ days for LSC. Performing concomitant hysterectomy at the time of apical prolapse repair did not significantly impact mean length of stay for all cohorts.

\section{Discussion}

The use of robotic-assisted surgery in apical prolapse repair is rapidly growing in the United States. With a 16-fold increase in the number of cases over the five-year study period, RSC comprised almost $25 \%$ of all minimally-invasive abdominal sacrocolpopexies by 2012. The study also demonstrated that RSC is the most expensive surgical approach to apical prolapse repair, and this finding is likely related to the additional cost of acquisition and maintenance of the robotic system and the recurring costs of consumables. Operating room fees are also more expensive with RSC, owing in part to increased staffing, additional time for robotic setup and docking, and prolonged operating times [17-20]. Direct costs related to anesthesia, hospital room, medication, imaging and laboratory studies have not been shown to be significantly different among various approaches to apical prolapse repair [16].

Significantly higher costs associated RSC compared to ASC, VC, and LSC were observed in both the index period and the follow-up period. This finding is consistent with trends observed in other costanalysis studies, although the difference in index cost observed in the current study of $\$ 3,420$ for ASC and $\$ 3,034$ for LSC fall somewhere between the cost difference cited in other studies. These studies report a mean cost difference ranging from $\$ 2,716$ to $\$ 11,011$ for ASC and $\$ 1,155$ to $\$ 8,043$ for LSC $[6,12-13,16]$. Variations in mean cost difference compared to this study may be related to small sample size, single institution experience, and/or exclusion of other direct costs 
Table 2. Complications and procedures of interest during the follow-up period

\begin{tabular}{|c|c|c|c|c|c|c|c|c|c|c|c|c|c|c|c|c|c|c|c|c|c|c|}
\hline & \multicolumn{4}{|c|}{ Robotic (Reference) } & \multicolumn{6}{|c|}{ Abdominal } & \multicolumn{6}{|c|}{ Vaginal } & \multicolumn{6}{|c|}{ Laparoscopic } \\
\hline & \multicolumn{2}{|c|}{$\begin{array}{l}\text { Without hysterectomy } \\
\qquad(\mathrm{N}=699)\end{array}$} & \multicolumn{2}{|c|}{$\begin{array}{l}\text { With hysterectomy } \\
\qquad(\mathrm{N}=1,307)\end{array}$} & \multicolumn{3}{|c|}{$\begin{array}{l}\text { Without hysterextomy } \\
\qquad(\mathrm{N}=2,555)\end{array}$} & \multicolumn{3}{|c|}{$\begin{array}{l}\text { With hysterectomy } \\
\qquad(\mathrm{N}=3,571)\end{array}$} & \multicolumn{3}{|c|}{$\begin{array}{l}\text { Without hysterectomy } \\
\qquad(\mathrm{N}=22,037)\end{array}$} & \multicolumn{3}{|c|}{$\begin{array}{l}\text { With hysterectomy } \\
(\mathrm{N}=23,771)\end{array}$} & \multicolumn{3}{|c|}{$\begin{array}{l}\text { Without hysterectomy } \\
\qquad(\mathrm{N}=2,651)\end{array}$} & \multicolumn{3}{|c|}{$\begin{array}{l}\text { With hysterectomy } \\
\qquad(\mathrm{N}=6,051)\end{array}$} \\
\hline & $\mathbf{N}$ & $\%$ & $\mathbf{N}$ & $\%$ & $\mathbf{N}$ & $\%$ & p-value & $\mathbf{N}$ & $\%$ & p-value & $\mathbf{N}$ & $\%$ & p-value & $\mathbf{N}$ & $\%$ & p-value & $\mathbf{N}$ & $\%$ & p-value & N & $\%$ & p-value \\
\hline \multicolumn{23}{|l|}{ Complication } \\
\hline Dyspareunia & 3 & $0.43 \%$ & 4 & $0.31 \%$ & 20 & $0.80 \%$ & 0.371 & 23 & $0.66 \%$ & 0.240 & 24 & $0.15 \%$ & 0.050 & 53 & $0.30 \%$ & 0.974 & 10 & $0.38 \%$ & 0.840 & 17 & $0.28 \%$ & 0.874 \\
\hline Perforation of intestine & 0 & $0.00 \%$ & 0 & $0.00 \%$ & 11 & $0.44 \%$ & 0.188 & 14 & $0.40 \%$ & 0.122 & 7 & $0.04 \%$ & 0.597 & 20 & $0.11 \%$ & 0.258 & 1 & $0.04 \%$ & 0.695 & $\mathbf{9}$ & $0.15 \%$ & 0.263 \\
\hline Stricture/kin king of ureter & 3 & $0.43 \%$ & 2 & $0.15 \%$ & 4 & $0.16 \%$ & 0.008 & 3 & $0.09 \%$ & 0.342 & 31 & $0.19 \%$ & 0.152 & 21 & $0.12 \%$ & 0.730 & 5 & $0.19 \%$ & 0.078 & 10 & $0.17 \%$ & 0.923 \\
\hline Other ureteric obstruction & 0 & $0.00 \%$ & 0 & $0.00 \%$ & 1 & $0.04 \%$ & 0.691 & 0 & $0.00 \%$ & 0.999 & 3 & $0.02 \%$ & 0.729 & 1 & $0.01 \%$ & 0.800 & 0 & $0.00 \%$ & 0.999 & 0 & $0.00 \%$ & 0999 \\
\hline Bladder \& urethra injury & 0 & $0.00 \%$ & 1 & $0.08 \%$ & 1 & $0.04 \%$ & 0.691 & 2 & $0.06 \%$ & 0.788 & 5 & $0.03 \%$ & 0.655 & 4 & $0.02 \%$ & 0.167 & 0 & $0.00 \%$ & 0.999 & 6 & $0.10 \%$ & 0819 \\
\hline Mechanical complication, implant/graft & 4 & $0.57 \%$ & 18 & $1.38 \%$ & 15 & $0.60 \%$ & 0.932 & 34 & $0.98 \%$ & 0.157 & 208 & $1.30 \%$ & 0.105 & 290 & $1.65 \%$ & 0.466 & 18 & $0.68 \%$ & 0.765 & 90 & $1.49 \%$ & 0.771 \\
\hline Other complications, implant/graft & 26 & $3.72 \%$ & 86 & $6.58 \%$ & 118 & $4.73 \%$ & 0.298 & 155 & $4.48 \%$ & $\$ .001$ & 806 & $5.02 \%$ & 0.136 & 1376 & $782 \%$ & 0.126 & 97 & $3.66 \%$ & 0.945 & 403 & $6.66 \%$ & 0.922 \\
\hline Embolus/phlebitis, not specified & 3 & $0.43 \%$ & 3 & $0.23 \%$ & $\mathbf{9}$ & $0.36 \%$ & 0.781 & 7 & $0.20 \%$ & 0.844 & 11 & $0.07 \%$ & $\$ .001$ & 16 & $0.09 \%$ & 0.087 & 4 & $0.15 \%$ & 0.004 & 7 & $0.12 \%$ & 0.198 \\
\hline DVI, lower extremities & 2 & $0.29 \%$ & 0 & $0.00 \%$ & 2 & $0.08 \%$ & 0.999 & 0 & $0.00 \%$ & 0.999 & 6 & $0.04 \%$ & $\$ .001$ & 3 & $0.02 \%$ & 0.661 & 6 & $0.23 \%$ & 0.757 & 6 & $0.10 \%$ & 0.361 \\
\hline Venous thrombosis, other veins & 22 & $3.15 \%$ & 33 & $253 \%$ & 124 & $497 \%$ & 0.074 & 118 & $3.41 \%$ & 0.172 & 866 & $5.39 \%$ & 0.013 & 679 & $386 \%$ & 0.020 & 164 & $6.19 \%$ & 0.008 & 244 & $4.03 \%$ & 0.019 \\
\hline Pneumonia as piration & 0 & $0.00 \%$ & 1 & $0.08 \%$ & 0 & $0.00 \%$ & 0999 & 0 & $0.00 \%$ & 0.999 & 4 & $0.02 \%$ & 0.690 & 2 & $0.01 \%$ & 0.004 & 0 & $0.00 \%$ & 0.999 & 2 & $0.03 \%$ & 0.326 \\
\hline Res piratory failure/arrest & 3 & $0.43 \%$ & 3 & $0.23 \%$ & 4 & $0.16 \%$ & 0.008 & 11 & $0.32 \%$ & 0.656 & 93 & $0.58 \%$ & 0.612 & 43 & $0.24 \%$ & 0.918 & 13 & $0.49 \%$ & 0.839 & 11 & $0.18 \%$ & 0.703 \\
\hline Other vascular complications & 46 & $6.58 \%$ & 69 & $5.28 \%$ & 223 & $893 \%$ & 0.080 & 258 & $7.45 \%$ & 0.024 & 1839 & $11.45 \%$ & $\$ .001$ & 1505 & $8.55 \%$ & $\$ .001$ & 244 & $9.21 \%$ & 0.052 & 381 & $6.30 \%$ & 0.195 \\
\hline Hemorrhage/hematoma & 4 & $0.57 \%$ & 8 & $0.61 \%$ & 8 & $0.32 \%$ & 0.212 & 14 & $0.40 \%$ & 0.224 & 141 & $0.88 \%$ & 0.402 & 79 & $0.45 \%$ & 0.388 & 20 & $0.76 \%$ & 0.635 & 20 & $0.33 \%$ & 0.066 \\
\hline Other postoperative infection & 3 & $0.43 \%$ & 9 & $0.69 \%$ & 50 & $2.00 \%$ & 0.023 & 58 & $1.68 \%$ & 0.044 & 90 & $0.56 \%$ & 0.652 & 125 & $0.71 \%$ & 0.930 & 19 & $0.72 \%$ & 0.454 & 32 & $0.53 \%$ & 0.451 \\
\hline Persistent pos toperative fis tula & 7 & $1.00 \%$ & 20 & $153 \%$ & 72 & $288 \%$ & 0.021 & 98 & $283 \%$ & 0.035 & 128 & $0.80 \%$ & 0.551 & 306 & $1.74 \%$ & 0.585 & 33 & $125 \%$ & 0.618 & 112 & $185 \%$ & 0.451 \\
\hline UT, not specified & 142 & $20.31 \%$ & 259 & $19.83 \%$ & 555 & $22.24 \%$ & 0.349 & 621 & $17.94 \%$ & 0.155 & 3956 & $24.63 \%$ & 0.025 & 3762 & $21.38 \%$ & 0.244 & 626 & $23.64 \%$ & 0.117 & 1095 & $18.10 \%$ & 0.177 \\
\hline Rupture of bladder, nontraumatic & 7 & $1.00 \%$ & 6 & $0.46 \%$ & 11 & $0.44 \%$ & 0.005 & 16 & $0.46 \%$ & 0.990 & 73 & $0.45 \%$ & 0.031 & 71 & $0.40 \%$ & 0.758 & 9 & $0.34 \%$ & 8.001 & 27 & $0.45 \%$ & 0.949 \\
\hline \multicolumn{23}{|l|}{ Procedure } \\
\hline Removal of pros thetic graft & 3 & $0.43 \%$ & 6 & $0.46 \%$ & 2 & $0.08 \%$ & 0.999 & 7 & $0.20 \%$ & $<0.001$ & 127 & $0.79 \%$ & 0.298 & 48 & $0.27 \%$ & 0.200 & 12 & $0.45 \%$ & 0.934 & 12 & $0.20 \%$ & 0.016 \\
\hline Destruction of vaginal kesion & 0 & $0.00 \%$ & 3 & $0.23 \%$ & 2 & $0.08 \%$ & 0.575 & 4 & $0.12 \%$ & 0.103 & 18 & $0.11 \%$ & 0.397 & 66 & $0.38 \%$ & 0.415 & 1 & $0.04 \%$ & 0.695 & 5 & $0.08 \%$ & 0.019 \\
\hline Revision of ureter & 3 & $0.43 \%$ & 6 & $0.46 \%$ & 0 & $0.00 \%$ & 0.999 & 1 & $0.03 \%$ & 0.999 & 4 & $0.02 \%$ & 0.999 & 12 & $0.07 \%$ & $<0.001$ & 8 & $0.30 \%$ & 0.555 & 14 & $0.23 \%$ & 0.069 \\
\hline Exploratory laparotomy & 0 & $0.00 \%$ & 3 & $0.23 \%$ & 3 & $0.12 \%$ & 0.492 & 1 & $0.03 \%$ & 0.999 & 6 & $0.04 \%$ & 0.625 & 7 & $0.04 \%$ & $<0.001$ & 1 & $0.04 \%$ & 0.695 & 3 & $0.05 \%$ & 0.999 \\
\hline Blood transfusion & 0 & $0.00 \%$ & 0 & $0.00 \%$ & 2 & $0.08 \%$ & 0.575 & 0 & $0.00 \%$ & 0.999 & 16 & $0.10 \%$ & 0.424 & 19 & $0.11 \%$ & 0.270 & 3 & $0.11 \%$ & 0.497 & 4 & $0.07 \%$ & 0.456 \\
\hline Percutaneous nephrostomy tube & 0 & $0.00 \%$ & 0 & $0.00 \%$ & 0 & $0.00 \%$ & 0.999 & 3 & $0.09 \%$ & 0.474 & 6 & $0.04 \%$ & 0.625 & 5 & $0.03 \%$ & 0.572 & 2 & $0.08 \%$ & 0.579 & 2 & $0.03 \%$ & 0.598 \\
\hline Uneteroneocystostomy & 1 & $0.14 \%$ & 0 & $0.00 \%$ & 0 & $0.00 \%$ & 0.999 & 1 & $0.03 \%$ & 0.680 & 1 & $0.01 \%$ & 0.999 & 4 & $0.02 \%$ & 0.613 & 1 & $0.04 \%$ & 0.999 & 1 & $0.02 \%$ & 0.709 \\
\hline Repair of bladder wound & 0 & $0.00 \%$ & 1 & $0.08 \%$ & 0 & $0.00 \%$ & 0999 & 0 & $0.00 \%$ & 0.999 & 2 & $0.01 \%$ & 0.778 & 3 & $0.02 \%$ & 0.061 & 3 & $0.11 \%$ & 0.497 & 1 & $0.02 \%$ & 0.999 \\
\hline
\end{tabular}

DVT deep vein thrombosis, UTl urinary tract infection 
in other studies. Additionally, in this study, the difference in index and follow-up costs were more pronounced when hysterectomy was concomitantly performed.

Cost and utilization data were collected for up to ninety days after surgery. Ninety days was selected as the cut-off for analyses, as this time frame is typically considered as the perioperative period for major surgical procedures and is when most surgery-related complications arise. In this study, healthcare utilization during the ninety-day followup period was generally higher with RSC. Statistical analysis revealed a significantly higher proportion of emergency room visits following RSC compared to LSC when performed without hysterectomy. Outpatient visits occurred more frequently following RSC compared to VC with hysterectomy, LSC overall, all POP procedures with hysterectomy, and all POP procedures overall. Although there was a trend toward lower readmission rates with RSC, the difference in proportion was statistically significant only when compared to LSC with hysterectomy and LSC overall. When comparing RSC to ASC, there was no significant difference in all healthcare utilization endpoints.

Increased healthcare utilization with RSC compared to other approaches may be partly related to higher rates of complication and additional procedures associated with RSC. In this study, statistically significant differences in complication rates were observed for ureteral stricture/kinking and mesh-related complications. Ureteral stricture or kinking, in particular, occurred more frequently with RSC than ASC. Although ureteral injury may result from hysterectomy or other concomitantly performed procedures, these cases occurred when hysterectomy was not performed; therefore, the complication most likely resulted from the sacrocolpopexy procedure itself. The higher rate of ureteral injury associated with the robotic approach highlights the influence of a learning curve and the need for adequate training with the robotic platform.

In terms of mesh-related complications, the analysis revealed higher complication rates with RSC than ASC. When hysterectomy was not concomitantly performed, however, mesh-related complication rates were similar between the two groups, suggesting that the observed differences may be influenced by performance of concurrent hysterectomy, which is a known risk factor for mesh complications [21]. Mesh-related complication rates were comparable between RSC and LSC with or without hysterectomy. This finding is consistent with results from other studies comparing RSC and LSC [20,22].

Published data have demonstrated lower rates of dyspareunia and higher rates of bladder injury with RSC $[18,22]$. However, these complications were not found to be statistically different in the current analysis. While significant differences were observed for complications, such as venous thromboembolic events or respiratory failure, these complications are not directly related to a sacrocolpopexy itself, but rather secondary to the postoperative state.

More procedures were performed postoperatively following RSC compared to other groups. While this may be a result of increased complication rates associated with RSC, direct causation cannot be definitively established from a retrospective analysis, and particularly when significant differences were observed only when sacrocolpopexy was concomitantly performed with hysterectomy. The latter observation might suggest that the need for additional procedures following RSC may be related to the performance of hysterectomy and not necessarily the apical prolapse procedure itself. One of the many purported advantages of RSC is decreased length of stay [5-6, 23-24]. Interestingly, while mean length of hospital stay in this study was consistently shorter following RSC compared to ASC, VC and LSC, the differences were not statistically significant.

The current study utilized a large national database that provides a robust set of longitudinally linked data and has been widely used to study healthcare utilization and outcomes. The database is particularly appropriate for the current analysis, as it includes cost information from inpatient services, outpatient services and pharmaceutical claims from multiple providers and facilities, and thereby yields more comprehensive results. Furthermore, cost calculations incorporate not only direct costs of surgery, but also costs associated with the postoperative period for up to ninety days. To our knowledge, this is the first study to report nationally representative cost estimates of robotic surgery in POP in the United States. The study also presents new findings on RSC utilization and outcomes across the United States.

The study is limited by its retrospective analysis. As with large national databases, clinical context or information cannot be extracted, and data entry may be subject to error. The database also includes mostly privately insured patients. The need for reduced operating time is more evident in private health structures compared to academic teaching institutions. Given such variation in practice, the study's reported cost difference between RSC and other approaches may be underestimated. The trend toward higher costs with RSC, however, is congruent with conclusions from referenced studies that were conducted at academic institutions $[6,12-13]$.

In conclusion, RSC is the most expensive surgical approach to apical prolapse repair during the first ninety days following surgery. In addition to significantly higher direct costs during the index and follow-up periods compared to other apical prolapse procedures, RSC is generally associated with higher rates of healthcare utilization during the first ninety days following surgery. In order to enhance the costeffectiveness and sustainability of RSC for POP surgery, large-scale studies on utilization- and cost-minimization strategies are needed.

\section{Authors' contribution}

LL Lua: Project development, data collection, data analysis and interpretation, manuscript writing, manuscript editing.

P Pathak: Data collection, statistical analysis.

V Dandolu: Project development, data analysis and interpretation, manuscript editing.

\section{Acknowledgments}

None

\section{Funding}

None

\section{Conflict of Interest}

The authors declare that they have no conflict of interest.

\section{References}

1. Wu JM, Hundley AF, Fulton RG, Myers ER (2009) Forecasting the prevalence of pelvic floor disorders in U.S. Women: 2010 to 2050. Obstet Gynecol 114:1278-1283. [Crossref]

2. Hudson CO, Northington GM, Lyles RH, Karp DR (2014) Outcomes of robotic sacrocolpopexy: a systematic review and meta-analysis. Female Pelvic Med Reconstr Surg 20: 252-260. [Crossref] 
3. Sung HH, Ko KJ, Suh YS, Ryu GH, Lee KS (2017) Surgical outcomes and safety of robotic sacrocolpopexy in women with apical pelvic organ prolapse. Int Neurourol $\mathrm{J}$ 21: 68-74. [Crossref]

4. American College of Obstetricians and Gynecologists (2015) Robotic surgery in gynecology, Committee Opinion No. 628. Obstet Gynecol 125: 760-767.

5. Paraiso MF (2014) Robotic-assisted laparoscopic surgery for hysterectomy and pelvic organ prolapse repair. Fertil Steril 102: 933-938. [Crossref]

6. Anger JT, Mueller ER, Tarnay C, Smith B, Stroupe K, et al. (2014) Robotic compared with laparoscopic sacrocolpopexy: a randomized controlled trial. Obstet Gynecol 123: 5-12. [Crossref]

7. Callewaert G, Bosteels J, Housmans S, Verguts J, Van Cleynenbreugel B, et al. (2016) Laparoscopic versus robotic-assisted sacrocolpopexy for pelvic organ prolapse: a systematic review. Gynecol Surg 13: 115-123. [Crossref]

8. Iavazzo C, Papadopoulou EK, Gkegkes ID (2014) Cost assessment of robotics in gynecologic surgery: a systematic review. J Obstet Gynaecol Res 40: 2125-2134. [Crossref]

9. Lee RK, Mottrie A, Payne CK, Waltregny D (2014) A review of the current status of laparoscopic and robot-assisted sacrocolpopexy for pelvic organ prolapse. Eur Urol 65: 1128-1137. [Crossref]

10. Carter-Brooks CM, Du AL, Bonidie MJ, Shepherd JP (2018) The impact of a dedicated robotic team on robotic-assisted sacrocolpopexy outcomes. Female Pelvic Med Reconstr Surg 24: 13-16. [Crossref]

11. Linder BJ, Occhino JA, Habermann EB, Glasgow AE, Bews KA, et al. (2018) A national contemporary analysis of perioperative outcomes of open versus minimally invasive sacrocolpopexy. J Urol 200: 862-867. [Crossref]

12. Paraiso MF, Jelovsek JE, Frick A, Chen CC, Barber MD (2011) Laparoscopic compared with robotic sacrocolpopexy for vaginal prolapse: a randomized controlled trial. Obstet Gynecol 118: 1005-1013. [Crossref]

13. Judd JP, Siddiqui NY, Barnett JC, Visco AG, Havrilesky LJ, et al. (2010) Costminimization analysis of robotic-assisted, laparoscopic, and abdominal sacrocolpopexy. J Minim Invasive Gynecol 17: 493-499. [Crossref]
14. Elliott CS, Hsieh MH, Sokol ER, Comiter CV, Payne CK, et al. (2012) Robot-assisted versus open sacrocolpopexy: a cost-minimization analysis. J Urol 187: 638-643. [Crossref]

15. Hoyte L, Rabbanifard R, Mezzich J, Bassaly R, Downes K (2012) Cost analysis of open versus robotic-assisted sacrocolpopexy. Female Pelvic Med Reconstr Surg 18: 335-339. [Crossref]

16. Patel M, O'Sullivan D, Tulikangas PK (2009) A comparison of costs for abdominal, laparoscopic, and robot-assisted sacral colpopexy. Int Urogynecol $J$ 20: 223-228. [Crossref]

17. Mueller ER (2013) Why complex pelvic organ prolapse should be approached abdominally. Curr Opin Urol 23: 317-322. [Crossref]

18. Oliver JL, Kim JH (2017) Robotic sacrocolpopexy-Is it the treatment of choice for advanced apical pelvic organ prolapse? Curr Urol Rep 18: 66. [Crossref]

19. Pan K, Zhang Y, Wang Y, Wang Y, Xu H (2016) A systematic review and meta-analysis of conventional laparoscopic sacrocolpopexy versus robot-assisted laparoscopic sacrocolpopexy. Int J Gynaecol Obstet 132: 284-291. [Crossref]

20. Tan-Kim J, Menefee SA, Luber KM, Nager CW, Lukacz ES (2011) Robotic-assisted and laparoscopic sacrocolpopexy: comparing operative times, costs and outcomes. Female Pelvic Med Reconstr Surg 17: 44-49. [Crossref]

21. Cundiff GW, Varner E, Visco AG, Zyczynski HM, Nager CW, et al. (2008) Risk factor for mesh/suture erosion following sacral colpopexy. Am J Obstet Gynecol 199: 688. [Crossref]

22. Unger CA, Paraiso MF, Jelovsek JE, Barber MD, Ridgeway B (2014) Perioperative adverse events after minimally invasive abdominal sacrocolpopexy. Am J Obstet Gynecol 211: 547. [Crossref]

23. Li H, Sammon J, Roghmann F, Sood A, Ehlert M, et al. (2014) Utilization and perioperative outcomes of robotic vaginal vault suspension compared to abdominal or vaginal approaches for pelvic organ prolapse. Can Urol Assoc J 8:100-106. [Crossref]

24. Geller EJ, Siddiqui NY, Wu JM, Visco AG (2008) Short-term outcomes of robotic sacrocolpopexy compared with abdominal sacrocolpopexy. Obstet Gynecol 112: 12011206. [Crossref]

Copyright: C2019 Lua LL. This is an open-access article distributed under the terms of the Creative Commons Attribution License, which permits unrestricted use, distribution, and reproduction in any medium, provided the original author and source are credited. 